\title{
Overcrowding and Its Association With Patient Outcomes in a Median-Low Volume Emergency Department
}

\author{
J. Laureano Phillips ${ }^{\mathrm{a}}$, Bradford E. Jackson ${ }^{\mathrm{b}, \mathrm{c}}$, Elizabeth L. Fagan ${ }^{\mathrm{d}, \mathrm{e}}$, Steven E. Arze ${ }^{\mathrm{e}}$, \\ Brenton Major ${ }^{\mathrm{d}}$, Nestor R. Zenarosa ${ }^{\mathrm{e}, \mathrm{f}}$, Hao Wang ${ }^{\mathrm{e}}$, f, g
}

\begin{abstract}
Background: Crowding occurs commonly in high volume emergency departments (ED) and has been associated with negative patient care outcomes. We aim to assess ED crowding in a median-low volume setting and evaluate associations with patient care outcomes.

Methods: This was a prospective single-center study from November 14, 2016 until December 14, 2016. ED crowding was measured every $2 \mathrm{~h}$ by three different estimation tools: National Emergency Department Overcrowding Score (NEDOCS); Community Emergency Department Overcrowding Score (CEDOCS); and Severely-overcrowding Overcrowding and Not-overcrowding Estimation Tool (SONET) categorized under six different levels of crowding (not busy, busy, extremely busy, overcrowded, severely overcrowded, and dangerously overcrowded). Crowding scores were assigned to each patient upon ED arrival. We evaluated the distributions of crowding and patient ED length of stay (ED LOS) across estimation tools. Accelerated failure time models were utilized to estimate time ratios and their corresponding 95\% confidence intervals comparing median LOS across levels of crowding within each estimation tool.
\end{abstract}

Results: This study comprised 2,557 patients whose median ED LOS was $150 \mathrm{~min}$. Approximately $2 \%$ of patients arrived during $2 \mathrm{~h}$ time intervals deemed overcrowded regardless of the crowding tool used. Median ED LOS increased with the increased level of ED crowd-

Manuscript submitted August 14, 2017, accepted August 31, 2017

${ }^{a}$ Office of Clinical Research, John Peter Smith Health Network, 1500 S. Main St., Fort Worth, TX 76104, USA

${ }^{\mathrm{b}}$ Center for Outcomes Research, John Peter Smith Health Network, 1500 S. Main St., Fort Worth, TX 76104, USA

'Department of Biostatistics and Epidemiology, UNT Health Science Center School of Public Health, Fort Worth, TX 76107, USA

dDepartment of Emergency Medicine, Baylor Scott \& White Medical Center at McKinney, 5252 W. University Dr., McKinney, TX 75071, USA

'Integrative Emergency Services, 13737 Noel Rd., Suite 1200, Dallas, TX 75240 , USA

fDepartment of Emergency Medicine, Integrative Emergency Services, John Peter Smith Health Network, 1500 S. Main St., Fort Worth, TX 76104, USA 'Corresponding Author: Hao Wang, Department of Emergency Medicine, Integrative Emergency Services, John Peter Smith Health Network, $1500 \mathrm{~S}$. Main St., Fort Worth, TX 76104, USA. Email: hwang01@jpshealth.org

doi: https://doi.org/10.14740/jocmr3165w ing and prolonged median ED LOS ( $>150 \mathrm{~min})$ occurred at ED of extremely busy status. Time ratios ranged from 1.09 to 1.48 for NEDOCS, 1.25 - 1.56 for CEDOCS, and 1.26 - 1.72 for SONET.

Conclusion: Overcrowding rarely occurred in study ED with median-low annual volume and might not be a valuable marker for ED crowding report. Though similar patterns of prolonged ED LOS occurred with increased levels of ED crowding, it seems crowding alerts should be initiated during extremely busy status in this ED setting.

Keywords: Emergency department; Crowding; Length of stay

\section{Introduction}

Emergency department (ED) crowding has become more common in recent years and overcrowding occurs more often in moderate to high volume EDs [1, 2]. It has been reported that ED overcrowding has a negative impact on patient care as well as ED operations such as prolonged ED length of stay (LOS), increased rates of patients eloping/left without being seen (LWBS), increased medical errors, and decreased patient satisfaction [3-5]. Most studies report that these negative patient care outcomes surge when EDs are at an overcrowded status. In a 2012 Emergency Department Benchmarking Alliance (EDBA) report, US median ED volume was approaching 40,000 visits/year, which is consistent with reports in the literature of overcrowding occurring more often in EDs with over 40,000 annual volume [6]. However, little is known about overcrowding at EDs below the national median volume and whether such overcrowding status should be considered a universal marker for negative patient care outcomes.

Although there are several ED crowding estimation tools reporting varying levels of crowding, each has different definitions of overcrowding, none of which is considered the "gold standard". Therefore, challenges persist in accurately determining the crowding status of different EDs. A review of the literature suggests that overcrowding is defined similarly across the following three ED crowding estimation tools: National Emergency Department Overcrowding Score (NEDOCS), Community Emergency Department Overcrowding Score (CEDOCS), and Severely-overcrowding Overcrowding and Not-overcrowding Estimation Tool (SONET). NEDOCS is by far the most 
widely used tool for ED crowding measurements [7]. However, NEDOCS was derived in the moderate to high volume ED setting and its ability to determine ED crowding in a median-low volume ED has not been validated. CEDOCS was derived in EDs ranging from low to high volume and has been deemed appropriate for crowding measurement across a wide range of ED annual volume but lacks external validation [8]. SONET was derived using similar methods as NEDOCS and validated externally at different EDs where the same group provides administrative operation and management at the study ED [9].

It is uncertain whether consistency could be reached among different tools in terms of overcrowding status in the median-low volume ED setting. Therefore, the primary aim of this study was to examine crowding at an ED of median-low volume using different estimation tools for consistency assessment. Additionally, we sought to evaluate the associations between crowding status and patient care outcomes.

\section{Methods}

\section{Study designs and participants}

This is a cohort study with prospective exposure-outcome assessment conducted at Baylor Scott \& White Medical Center at McKinney from 8 am on November 14, 2016 until 8 am on December 14, 2016. The study time frame was selected purely on study convenience. Baylor Scott \& White Medical Center at McKinney is a community hospital with a total of 143 licensed beds. The study ED has 23 beds with patient annual visits of approximately 32,000 - 35,000 over the past 3 years. It is a level 3 trauma center, but not a designated transplant center. The average admission rate of the study ED over the past 3 years was below 20\%. Taken together, the study ED is considered low acuity with a median-low volume as defined by EDBA [10]. Eligible study participants included all patients who registered at the ED as a patient upon arrival during the study period. Each patient was assigned crowding scores determined by different ED crowding tools as described in more detail in subsection titled, "ED crowding scores". Patients for whom crowding scores could not be assigned were excluded from the final data analysis. The Baylor Scott \& White Institutional Review Board approved this study.

\section{Study protocol}

The purpose of this study was to evaluate the frequency of crowding status and its association with patient care outcomes. Specifically, this study sought to determine whether overcrowding was associated with similar poor patient outcomes as reported in moderate to high volume EDs. Briefly, three different crowding scores were assigned to each patient upon their ED arrival. Median ED LOS of all patients was measured and used as an additional operational marker. Patients whose ED LOSs were beyond this marker were considered prolonged ED stay. ED outcomes (e.g. ED LOS and ED LWBS rate) were measured and compared under different levels of ED crowding using each of the three different estimation tools.

\section{ED crowding scores}

ED crowding scores were calculated independently with three estimation tools (NEDOCS, CEDOCS, and SONET). All three tools can determine ED crowding with a continuous score. NEDOCS and CEDOCS scores can be calculated within the same ranges and subdivided into six distinct levels (not busy $(0$ - 20), busy (21 - 60), extremely busy (61 - 100), overcrowded (101 - 140), severely overcrowded (141 - 180), and dangerously overcrowded (181 and above)) [7,8]. Since SONET was derived similarly to NEDOCS with the same score ranges, it is easily modified into the same six categories for comparison. More importantly, overcrowding is defined as crowding score beyond 100 in all three tools. NEDOCS and CEDOCS crowding scores were calculated online, while SONET scores were calculated internally using a pre-formulated excel sheet. A detailed description of scoring formulas and the variables required to calculate are shown in Supplementary Table (www. jocmr.org). ED crowding was measured at the top of every $2 \mathrm{~h}$ interval throughout the study period. Crowding scores estimated at the beginning of each interval were applied to all subjects who registered during that specific $2 \mathrm{~h}$ interval (e.g., patients who arrived in the ED from 0800 to 0900 were assigned to the same crowding score measured at 0800 and patients who arrived at ED from 1000 to 1159 were assigned to the same crowding score measured at 1000, and so on). In other words, each patient was assigned three different crowding scores (one for each of the crowding estimation tools used) upon arrival in the ED at the top of each $2 \mathrm{~h}$ interval.

\section{Patient outcome measurements}

Both ED LOS (measured in minutes) and eloped/LWBS outcomes demonstrate reliable correlation with ED crowding regardless of crowding tool and are frequently measured and reported in the literature [11-13]. However, in the present study, we observed less than $0.5 \%$ of patients were classified as LWBS. Due to the low frequency of LWBS, it was not considered as a primary outcome of interest. LWBS was defined as left before or after medical screening, left without provider evaluation or treatment, but does not include patients who left against medical advice. The primary patient outcome of interest for this study was ED LOS, defined as the total time in minutes from when the patient arrived at the ED until the patient physically left the ED, where patients were censored if they eloped/ LWBS. For eloped/LWBS patients, every $20 \mathrm{~min}$, three separate calls were rendered. If no response was received, patients were designated as eloped/LWBS and the first call time was documented as the time when the patient physically left the ED.

\section{Data analysis}

Patient characteristics are presented as: frequencies and percent- 
Table 1. Emergency Department Study Patient General Information

\begin{tabular}{|ll}
\hline Total number of patients registered at study ED (n) & 2,987 \\
Number of patients for data analysis (n) & 2,557 \\
Male (n, \%) & $1,066(42 \%)$ \\
Age (years) (median, IQR) & $42(25-63)$ \\
Acuity level (n, \%) & \\
ESI 1 & $13(0.51 \%)$ \\
ESI 2 & $290(11.34 \%)$ \\
ESI 3 & $1,566(61.24 \%)$ \\
ESI 4 & $609(23.82 \%)$ \\
ESI 5 & $52(2.03 \%)$ \\
Unclassified & $27(1.06 \%)$ \\
Admissions (n, \%) & $267(10.63 \%)$ \\
Length of stay (minutes) (median, IQR) & $150(99-213)$ \\
Patients eloped/LWBS (n, \%) & $8(0.3 \%)$ \\
\hline
\end{tabular}

$\mathrm{n}$ : number; IQR: interquartile range; ESI: emergency severity index; LWBS: left without being seen/eloped.

ages for categorical data; and medians and interquartile ranges (IQR) for continuous data. Box and whisker plots were generated to illustrate the distribution of ED LOS across crowding levels for each crowding assessment tool. Outlying ED LOS patients were included in the estimation of the median and IQRs, however, excluded from the graphical presentation in order to enhance visibility. For each crowding estimation tool, we report the frequency and percent of study time classified at different crowding levels; likewise, we estimated stratum specific Kaplan-Meier median (IQR) estimates of ED LOS. We performed non-parametric tests for trend across the levels of crowding [14].

In order to evaluate the associations between crowding status and ED LOS, we specified accelerated failure time models using the log-logistic distribution with robust variance to estimate time ratios (TRs) and their corresponding 95\% confidence intervals (CIs) $[15,16]$. Accelerated failure time models are parametric time-to-event models which provide more concise summarization of time-to-event data relative to the Cox regression model which assumes proportional hazards. The TR is interpreted as the median ED LOS relative to the reference category within each scoring system. In our study, not busy was the reference category for all estimation tools. The loglogistic distribution was selected based on goodness of fit tests using Akaike's information criterion to compare various distributional assumptions [17]. Stata version 14.2 (Stata Corp, College Station, TX) was used for the analyses.

\section{Results}

Table 1 summarizes study participant characteristics. A total of 2,987 patients presented and arrived at the ED between November 14, 2016 and December 14, 2016. Among these patients, 430 were not assigned crowding scores upon arrival due to incomplete data for real-time crowding score calculations and thus were excluded. Further investigation revealed that these missing data occurred in 3 days ( 8 am -8 pm) and two nights (8 pm - 8 am) when ED unit clerks were not notified to collect data. Our analytic sample thus comprised 2,557 patients with an $86 \%$ data completion rate. In brief, the study population included more females, where $42 \%$ were male. The median age at $\mathrm{ED}$ arrival was 42 years $(\mathrm{IQR}=25-63)$. In terms of level of acuity, over $60 \%$ of subjects were classified as emergency severity index 3 (ESI 3 ) patients and approximately $10 \%$ of patients were hospital admissions. The median ED LOS was 150 min and only eight $(0.3 \%)$ were classified as eloped/LWBS.

In this study, there were no observations of the ED being measured as severely overcrowded or dangerously overcrowded for any of the estimation tools. Table 2 presents the estimation tool specific frequencies and percentages of crowding as well as the Kaplan-Meier estimated median ED LOS and IQR. NEDOCS estimated the study ED as not busy more often than CEDOCS or SONET (NEDOCS $=34.42 \%$; CEDOCS $=$ $1.72 \%$; SONET $=1.56 \%$ ). Across all three estimation tools, busy was the most frequently classified category (NEDOCS $=43.25 \%$; $\mathrm{CEDOCS}=86.27 \%$; $\mathrm{SONET}=67.66 \%)$. The CEDOCS estimation tool did not classify any time interval as overcrowded. We observed statistically significant trends in median ED LOS across crowding level within each estimation tool $(\mathrm{P}<0.001)$. Meanwhile, median ED LOS increased with the increased level of ED crowding and prolonged median LOS ( $>150 \mathrm{~min}$ ) occurred at ED of extremely busy status regardless of crowding tool employed (Fig. 1).

TRs and their corresponding 95\% CIs comparing median ED LOS across levels of crowding within each estimation tool are presented in Table 3. With not busy as the reference

Table 2. Median Length of Stay in Minutes Across Levels of Crowding Measured by Different ED Crowding Tools

\begin{tabular}{|c|c|c|c|c|c|c|c|c|c|c|c|c|}
\hline & \multicolumn{4}{|c|}{ NEDOCS } & \multicolumn{4}{|c|}{ CEDOCS } & \multicolumn{4}{|c|}{ SONET } \\
\hline & $\mathbf{n}$ & $\%$ & $\begin{array}{l}\text { Median } \\
\text { LOS }\end{array}$ & IQR & $\mathbf{n}$ & $\%$ & $\begin{array}{l}\text { Median } \\
\text { LOS }\end{array}$ & IQR & $\mathbf{n}$ & $\%$ & $\begin{array}{l}\text { Median } \\
\text { LOS }\end{array}$ & IQR \\
\hline Busy & 1,106 & 43.3 & 150.6 & $98.6-207.8$ & 2,206 & 86.3 & 146.0 & $96.3-209.0$ & 1,730 & 67.7 & 142.4 & $93.3-204.8$ \\
\hline Extremely busy & 514 & 20.1 & 171.9 & $118.1-237.8$ & 307 & 12.0 & 185.6 & $124.9-252.0$ & 733 & 28.7 & 166.8 & $112.7-231.2$ \\
\hline
\end{tabular}

NEDOCS: National Emergency Department Overcrowding Score; CEDOCS: Community Emergency Department Overcrowding Score; SONET: Severely-overcrowding Overcrowding, and Not-Overcrowding Estimation Tool; LOS: length of stay; IQR: interquartile range. 


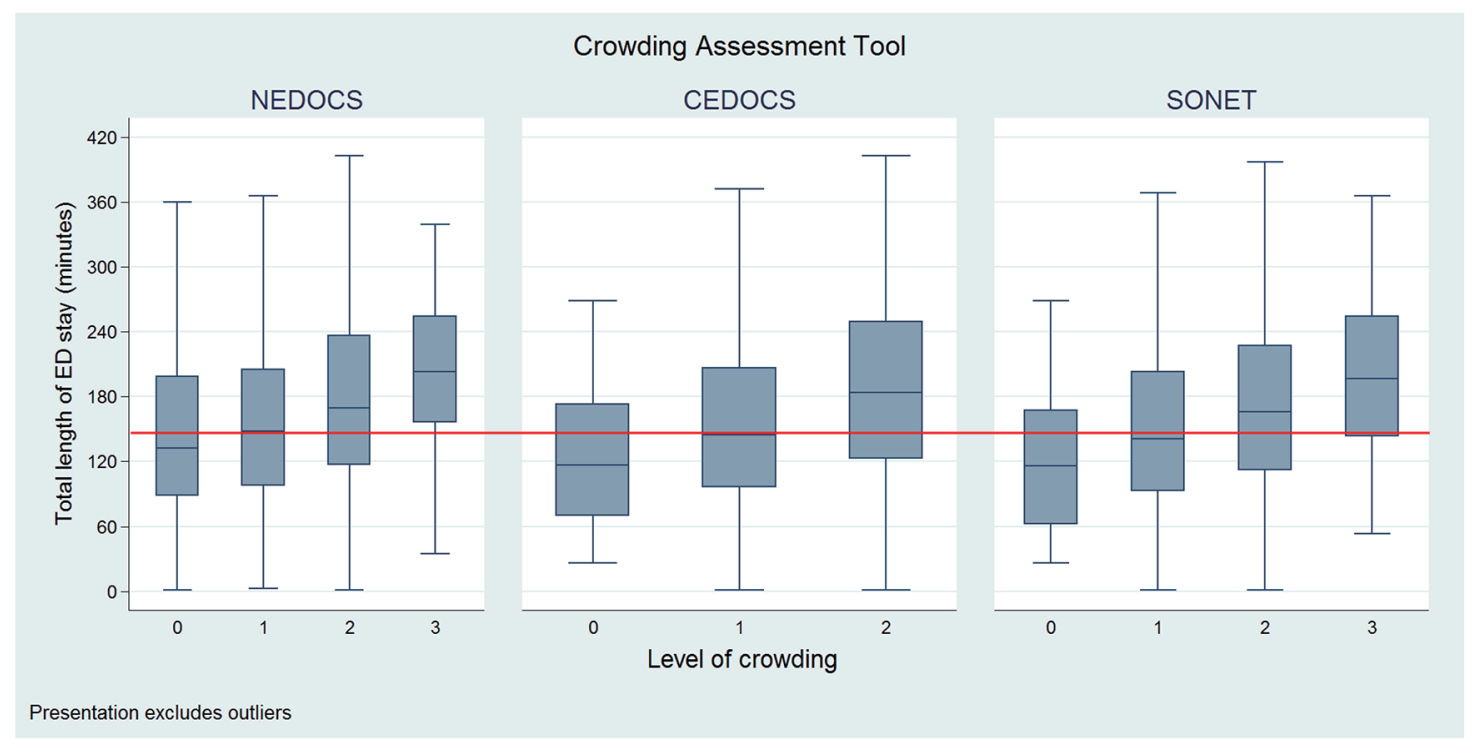

Figure 1. Boxplot of median total length of stay in patients under different levels of crowding measured by different crowding tools. The red line indicates the median length of stay (150 min) among all ED patients. 0: not busy; 1: busy; 2: extremely busy; 3: overcrowded.

category, we observed increased TR as the level of crowding increased with point estimates suggesting longer median ED LOS. Point estimates ranged from 1.09 to 1.48 for NEDOCS, from 1.25 to 1.56 for CEDOCS, and from 1.26 to 1.72 for SONET, where estimates for busy and extremely busy were similar in magnitude for both CEDOCS and SONET.

Though very few patients eloped/LWBS in this study, there was a higher percentage of eloped/LWBS with the increased level of crowding regardless of the estimation tool used. It was observed that higher eloped/LWBS occurred when the study ED was under extremely busy status, where $50 \%$ or more of those who eloped/LWBS did so when the ED was classified as extremely busy (Supplementary Figure, www.jocmr.org). As mentioned above, due to the relatively low frequency of eloped/LWBS patients, it was not considered as a reliable outcome measure in this study.

\section{Discussion}

ED crowding has become more common in the US and con- tinues to be associated with negative patient care outcomes $[1,18]$. Overcrowding occurs more commonly in EDs of high volume with less concerns in those with low volume. Our results show that, though there were some discrepancies upon the degree of patient distribution under ED of not overcrowded status, consistency with very few patients arriving under the overcrowded ED conditions occurred regardless of estimation tool employed (Table 2). This suggests that overcrowding is uncommon at EDs of median-low volume which subsequently raises the question of whether overcrowding is a valuable marker for ED crowding reporting. Interestingly, prolonged ED LOS occurred consistently with increased levels of ED crowding as determined by all three estimation tools. If the overall patient median ED LOS (150 min) was used as an indicator, prolonged median ED LOS occurred when ED crowding shifted from busy to extremely busy status, despite of estimation tool. This indicates that action plans to improve patient flow should be implemented at median-low volume EDs at or before an extremely busy status. It might be too late to initiate efficiency operation strategies under ED overcrowded status. Although previous studies showed prolonged ED LOS associ-

Table 3. Time Ratios for Median Length of Stay Across Levels of Crowding Measured by Different ED Crowding Tools

\begin{tabular}{|c|c|c|c|c|c|c|}
\hline & \multicolumn{2}{|c|}{ NEDOCS } & \multicolumn{2}{|c|}{ CEDOCS } & \multicolumn{2}{|c|}{ SONET } \\
\hline & TR & $95 \%$ CI & TR & $95 \%$ CI & TR & $95 \%$ CI \\
\hline Not busy & REF & REF & REF & REF & REF & REF \\
\hline Busy & 1.09 & $1.04-1.15$ & 1.25 & $1.01-1.55$ & 1.26 & $0.99-1.61$ \\
\hline Extremely busy & 1.27 & $1.19-1.35$ & 1.56 & $1.25-1.95$ & 1.47 & $1.15-1.87$ \\
\hline Overcrowded & 1.48 & $1.32-1.67$ & - & - & 1.72 & $1.31-2.25$ \\
\hline
\end{tabular}

NEDOCS: National Emergency Department Overcrowding Score; CEDOCS: Community Emergency Department Overcrowding Score; SONET: Severely-overcrowding Overcrowding, and Not-Overcrowding Estimation Tool; TR: time ratio; Cl: confidence interval; REF: reference. 
ated with increased levels of ED crowding, mainly in moderate to high volume ED settings $(4,13)$, our results provide extra support, expanding the association to include median-low volume EDs as well. These findings may be utilized to further help ED administrators manage patient flow more efficiently in EDs of median-low volume.

Our study used three different estimation tools to determine ED crowding. NEDOCS was derived at moderate to high volume EDs and has been validated in other studies [19, 20]. Crowding and its association with negative patient outcomes, including prolonged ED LOS and increased eloped/ LWBS rates, have been consistently reported in the literature [21]. However, these methods may not be suitable as crowding measurements at median-low volume ED settings. CEDOCS was derived at community EDs and reported to be able to determine ED crowding at different settings including EDs of low, moderate, and high volumes, but lack of external validation [8]. SONET was derived and validated at extremely high volume EDs with the same healthcare ED group and operation system used in this study, has not been externally validated for use in median-low volume EDs [9]. However, the intent of this study was not to compare the accuracy of each ED crowding estimation tool, but rather to use each to determine if estimation consistency can be reached. Our results showed high consistency among all three tools when assigning overcrowding status. Moreover, high consistency was reached for the occurrence of negative patient care outcomes when ED crowding shifted from busy to extremely busy statuses. Since there is currently no "gold standard" to determine the accuracy of ED crowding, analyzing consistency among different crowding tools, our study will add value for future ED crowding research.

EDs classified as overcrowded operate at maximum capacity; therefore, administrators should begin to mobilize additional resources. This overcrowded level of ED crowding is relatively common in moderate to high volume ED settings. When using ED LOS and LWBS rates as outcome measurements, previous studies showed prolonged ED LOS and increased LWBS rates with increased levels of ED crowding when overcrowded status was reached [13, 21]. However, this approach may be inappropriate at EDs with median-low volume settings. Our study showed negative patient care outcomes (i.e., prolonged ED LOS, increased eloped/LWBS) shifted earlier in median-low volume EDs under an extremely busy status, indirectly pointing to the necessity of reporting ED crowding earlier at EDs of median-low volume in comparison to high volume EDs.

\section{Limitations}

Results of this study should be considered in light of certain limitations. Notably, this study was a single-center prospective study with potential selection bias. Our study enrolled $86 \%$ of patients during the study period; therefore, we are unable to determine outcomes for the remaining $14 \%$ who were not enrolled. However, it should be noted that excluded subjects did not differ from the analysis population in terms of age, sex, and acuity (data not shown). Secondly, study time frame was selected at convenience but not randomly which could have potential time selection bias. In addition to this, given the focus of our study on three different crowding tools, the generalizability of our results to other tools is largely unknown. Though ED LOS and eloped/LWBS rates are among the most common patient care outcome metrics reported in routine ED operations, other outcomes (e.g., $72 \mathrm{~h}$ ED return, patient satisfaction, etc.) are not reported in this study. As we observed very few patients designated as eloped/LWBS, we were unable to evaluate associations with this outcome. Future multi-center prospective studies in median-low volume EDs with large sample sizes are warranted to further validate our findings.

\section{Conclusion}

In conclusion, although overcrowding rarely occurred in the current median-low volume ED, our study showed similar negative patient outcomes occurred with increased levels of ED crowding. We recommend that in median-low volume ED settings, crowding reports/alerts should be initiated earlier when the ED is classified as extremely busy instead of overcrowded statuses.

\section{Funding}

None.

\section{Conflict of Interest}

None.

\section{Author Contributions}

HW conceived the study and designed the protocol. HW, JLP, ELF, SEA, and BM performed the study and data collection. HW, JLP, and BEJ performed the data analysis. HW, JLP, BEJ, and NRZ drafted the article, and all authors contributed substantially to this study. HW takes responsibility for the paper as a whole.

\section{References}

1. Eitel DR, Rudkin SE, Malvehy MA, Killeen JP, Pines JM. Improving service quality by understanding emergency department flow: a White Paper and position statement prepared for the American Academy of Emergency Medicine. J Emerg Med. 2010;38(1):70-79.

2. Olshaker JS. Managing emergency department overcrowding. Emerg Med Clin North Am. 2009;27(4):593603, viii.

3. Epstein SK, Huckins DS, Liu SW, Pallin DJ, Sullivan AF, Lipton RI, Camargo CA, Jr. Emergency department crowding and risk of preventable medical errors. Intern Emerg Med. 2012;7(2):173-180. 
4. Bernstein SL, Aronsky D, Duseja R, Epstein S, Handel D, Hwang U, McCarthy M, et al. The effect of emergency department crowding on clinically oriented outcomes. Acad Emerg Med. 2009;16(1):1-10.

5. Carter EJ, Pouch SM, Larson EL. The relationship between emergency department crowding and patient outcomes: a systematic review. J Nurs Scholarsh. 2014;46(2):106-115.

6. Welch SJ, Augustine JJ, Dong L, Savitz LA, Snow G, James BC. Volume-related differences in emergency department performance. Jt Comm J Qual Patient Saf. 2012;38(9):395-402.

7. Weiss SJ, Derlet R, Arndahl J, Ernst AA, Richards J, Fernandez-Frackelton M, Schwab R, et al. Estimating the degree of emergency department overcrowding in academic medical centers: results of the National ED Overcrowding Study (NEDOCS). Acad Emerg Med. 2004;11(1):3850 .

8. Weiss SJ, Rogers DB, Maas F, Ernst AA, Nick TG. Evaluating community ED crowding: the Community ED Overcrowding Scale study. Am J Emerg Med. 2014;32(11):1357-1363.

9. Wang H, Robinson RD, Garrett JS, Bunch K, Huggins CA, Watson K, Daniels J, et al. Use of the SONET Score to Evaluate High Volume Emergency Department Overcrowding: A Prospective Derivation and Validation Study. Emerg Med Int. 2015;2015:401757.

10. Welch S, Augustine J, Camargo CA, Jr., Reese C. Emergency department performance measures and benchmarking summit. Acad Emerg Med. 2006;13(10):1074-1080.

11. Pines JM, Prabhu A, Hilton JA, Hollander JE, Datner EM. The effect of emergency department crowding on length of stay and medication treatment times in discharged patients with acute asthma. Acad Emerg Med. 2010;17(8):834-839.

12. Kulstad EB, Hart KM, Waghchoure S. Occupancy rates and emergency department work index scores correlate with leaving without being seen. West J Emerg Med.
2010;11(4):324-328.

13. McCarthy ML, Zeger SL, Ding R, Levin SR, Desmond JS, Lee J, Aronsky D. Crowding delays treatment and lengthens emergency department length of stay, even among high-acuity patients. Ann Emerg Med. 2009;54(4):492503 e494.

14. Cuzick J. A Wilcoxon-type test for trend. Stat Med. 1985;4(1):87-90.

15. Lee ET, Go OT. Survival analysis in public health research. Annu Rev Public Health. 1997;18:105-134.

16. Chaou CH, Chiu TF, Yen AM, Ng CJ, Chen HH. Analyzing Factors Affecting emergency department length of stay-using a competing risk-accelerated failure time model. Medicine (Baltimore). 2016;95(14):e3263.

17. Akaike H. A New look at the statistical identification. IEEE Trans Automat Contr. 1974;19(6):716-723.

18. Casalino E, Choquet C, Bernard J, Debit A, Doumenc B, Berthoumieu A, Wargon M. Predictive variables of an emergency department quality and performance indicator: a 1-year prospective, observational, cohort study evaluating hospital and emergency census variables and emergency department time interval measurements. Emerg Med J. 2013;30(8):638-645.

19. Boyle A, Abel G, Raut P, Austin R, Dhakshinamoorthy V, Ayyamuthu R, Murdoch I, et al. Comparison of the International Crowding Measure in Emergency Departments (ICMED) and the National Emergency Department Overcrowding Score (NEDOCS) to measure emergency department crowding: pilot study. Emerg Med J. 2016;33(5):307-312.

20. Hoot N, Aronsky D. An early warning system for overcrowding in the emergency department. AMIA Annu Symp Proc. 2006:339-343.

21. Weiss SJ, Ernst AA, Derlet R, King R, Bair A, Nick TG. Relationship between the National ED Overcrowding Scale and the number of patients who leave without being seen in an academic ED. Am J Emerg Med. 2005;23(3):288-294. 